\title{
Location and classification of Canalis sinuosus for cone beam computed tomography: avoiding misdiagnosis
}

\begin{abstract}
Luiz Roberto Coutinho MANHÃES JÚNIOR(a) Maria Fernanda Lima VILLAÇA-CARVALHO(b) Mari Eli Leonelli de MORAES(a) Sérgio Lúcio Pereira de Castro LOPES $^{(a)}$

Milena Bortolotto Felippe SILVA(c) José Luiz Cintra JUNQUEIRA(c)
\end{abstract}

\footnotetext{
(a) Universidade Estadual Paulista - Unesp, Institute of Science and Technology, Department of Oral Radiology, São José dos Campos, SP, Brazil.

(b) Universidade Estadual Paulista - Unesp, Institute of Science and Technology, Department of Oral Pathology, São José dos Campos, SP, Brazil.

(c)Faculdade São Leopoldo Mandic - SLM, School of Dentistry, Department of Radiology, Campinas, SP, Brazil.
}

Declaration of Interests: The authors certify that they have no commercial or associative interest that represents a conflict of interest in connection with the manuscript.

Corresponding Author: Maria Fernanda Lima Villaça-Carvalho E-mail: mfervillaca@hotmail.com

DOI: 10.1590/1807-3107BOR-2016.vol30.0049

Submitted: Aug 12, 2015

Accepted for publication: Dec 18, 2015

Last revision: Feb 20, 2016

\begin{abstract}
The aim of this study was to assess the presence, location and, multiplanar distance of the canalis sinuosus (CS) between the incisive foramen and the anterior maxillary alveolar ridge using cone beam computed tomography (CBCT). Therefore, 500 CBCT maxillary images obtained from male and female patients aged 20 to 80 years were selected to assist in the dental treatment. Low-quality tomographic images were discarded. All images were captured with the $\mathrm{i}-\mathrm{CAT}^{\mathrm{TM}}$ Classic tomograph and assessed using the $\mathrm{XoranCat}^{\mathrm{TM}}$ software. The axial sections were analyzed at the incisive foramen in order to verify the CS presence in laterality and location. Furthermore, linear measurements of the nasal cavity floor, buccal cortical bone, and alveolar ridge crest were made. All the collected data were statistically analyzed. Results show a variation of the CS in relation to the classification and distance of anatomical structures, but no significant difference between the right and left sides. It should be highlighted that $\mathrm{CBCT}$ is necessary before invasive procedures in order to preserve important anatomical structures. In conclusion, the location of the CS varies in relation to the alveolar ridge crest and buccal cortical bone, assuming that it is going to be located by the upper lateral incisor palatine.
\end{abstract}

Keywords: Cone Beam Computed Tomography; Diagnosis; Pathological Conditions, Anatomical.

\section{Introduction}

The canalis sinuosus (CS) is a neurovascular canal, where the anterior superior alveolar nerve and its blood vessels are located, that is not well understood by oral surgeons. It was first suggested by Jones, ${ }^{1}$ who described this anatomical structure as the nerve and blood vessels that leave the infraorbital nerve through the back part of the infraorbital foramen and run laterally through a bone canal of about $2 \mathrm{~mm}$ in diameter beside the nasal cavity. ${ }^{2}$

The infraorbital nerve is a direct extension of the maxillary nerve and the second division of the trigeminal nerve (fifth cranial nerve - $\mathrm{CN} \mathrm{V}$ ), which is related to the sensitivity of the skin and mucosa of the middle third of the face. ${ }^{3}$ A small branch is issued by the infraorbital canal on its side, close to the midpoint, to allow the passage of the anterior superior alveolar nerve. This canal, sometimes called the CS, runs to the 
anterior and inferior wall of the orbital cavity, beside the infraorbital canal and medially bent toward the anterior wall of the maxillary sinus, passing below the infraorbital foramen; it is a rare anatomical variation of the anterior superior alveolar nerve, which is a major concern in any surgical procedure such as surgery in the maxillary sinus, orthognathic surgery, and mostly in the placement of implants. ${ }^{4,5}$

According to CBCT guidelines, ${ }^{6}$ it is clear that CBCT has been widely used in implant dentistry. As such, we propose that CBCT should be indicated for crosssectional imaging prior to implant placement as an alternative to existing cross-sectional techniques in which the radiation dose of CBCT is shown to be lower. In the case of cross-sectional imaging prior to implant placement, CBCT offers the advantage of adjustable fields of view.

The identification of these anatomical variations has become more evident with the use of CBCT in dentistry because of the quality and accuracy of this type of examination. In the past, these morphological changes were hardly identified as a result of poor-quality imaging scans, which could cause intraoperative complications of unknown etiology during a surgical procedure in the anterior maxillary region, as indicated by Faria et al., ${ }^{7}$ who emphasized that examination, diagnosis, and proper treatment planning can prevent iatrogenic incidents such as inadvertent infraorbital nerve incision, which would cause permanent facial paresthesia. Barnett et al. ${ }^{8}$ also pointed out that infraorbital nerve damage is one of the most frequent complications of Caldwell-Luc surgery, leading to facial paresthesia.

However, with technological advancements, especially regarding the use of $\mathrm{CBCT}$, the amount of information obtained through images has increased and the diagnosis of these variations has become more evident. ${ }^{9,10}$

Although the opening in the anterior palate is a normal anatomical aspect, it is a variation that has not been properly described, thus requiring special attention in surgical procedures. ${ }^{11}$ CBCT is then indicated because it provides precise details of the studied region and eliminates overlapping images, in addition to collecting reliable linear and angular measurements and allowing reconstruction of the image in several planes and in 3D. ${ }^{12}$

Thus, the aim of this study was to assess the presence, location, and multiplanar distance of the CS between the incisive foramen and the anterior maxillary alveolar ridge using CBCT.

\section{Methodology}

\section{Sample}

This non-experimental research, which made use of convenience sampling, was approved by the Research Ethics Committee of the Instituto de Ciência e Tecnologia de São José dos Campos - ICT-Unesp (protocol XFSR/2013). This was a retrospective study and no patients were exposed solely for research purposes.

Based on Oliveira-Santos et al. ${ }^{11}$ and on Temmerman et al., ${ }^{13} 500$ CBCT images were used, which belong to the archives of the Department of Radiology of Unesp, São José dos Campos, and to Tomoface, a private clinic in São José dos Campos, SP, Brazil. Therefore, according to the seven emergence possibilities described by Oliveira-Santos et al., ${ }^{11}$ the foramina and additional canals in the anterior palate region were classified, and the distance in which they emerge in relation to the nasal cavity floor, the ridge's buccal cortical bone and the alveolar ridge crest was measured through the use of CBCT.

The following inclusion criteria apply to this study: images from the archives of the Department of Radiology of UNESP and of Tomoface; male and female subjects aged 20 to 80 years; and patients who had their maxillae assessed by CBCT. Patients who fell into the following criteria were excluded: those who had undergone surgical procedures or bone grafting in the anterior maxilla; those presenting pathological lesions or trauma (plate and screws) in the anterior maxilla; and those whose CT scans failed to present satisfactory quality.

\section{Equipment}

All CBCT images were obtained with I-Cat ${ }^{\mathrm{TM}}$ Classic (Imaging Sciences International, Hatfield, USA), with a standardized voxel of $0.25 \mathrm{~mm}$, field of view (FOV) of $8 \mathrm{~cm}$, and acquisition time 
of $40 \mathrm{~s}$ (Hi-Resolution). The radiation time was shorter because the images were acquired by pulse sequences. The default settings of the equipment were used, with the tube voltage kept constant at $120 \mathrm{kV}$ and a variation of 5 to $7 \mathrm{~mA}$, depending on the selected resolution. All images were processed and edited by the XoranCat ${ }^{\mathrm{TM}}$ software (Xoran Technologies, Ann Arbor, USA), with minor brightness and contrast corrections to make their visualization easier. The "Angio-Sharpen-Low $3 \times 3$ " image filter was applied for the sake of standardization and so that the tomographic sections could show sufficient anatomical details for the identification of the CS. In order to standardize the measurements and the classification of the CS, $0.25 \mathrm{~mm}$ axial sections and cross-sections with thickness and length of $1.00 \mathrm{~mm}$ were used.

\section{Image editing}

As soon as the CBCT images were produced by the XoranCat ${ }^{\mathrm{TM}}$ software, it was possible to retrospectively evaluate the patients' anterior maxillae. On the multiplanar reconstruction screen (MPR), the anatomical axial, coronal, and sagittal planes were aligned. For the axial images, the plates of the pterygoid processes of the sphenoid bone were aligned with the coronal plane, thus aligning the anterior nasal spine with the sagittal plane. As for the sagittal images, the maxillary plane was used as reference so that the plane between the anterior nasal spine and the posterior nasal spine was parallel to the horizontal or axial plane. Finally, in the coronal sections, the hard palate and the nasal cavity floor were aligned with the horizontal or axial plane. For evaluations and measurements, the images generated by the multiplanar reconstruction in the axial, sagittal, coronal, and transverse planes were analyzed by the specific tools of the CBCT software.

\section{CS classification}

Once the edited axial sections were positioned correctly, the upper-lower direction of the incisive foramen region was scanned from near the midline to the upper canine. This analysis was carried out to verify the presence of the CS.
When the presence of the CS was observed, the seven emergence possibilities, according to their location relative to the teeth/incisive foramen (central incisor region, region between the central and lateral incisors, lateral incisor region, canine region, first premolar region, lateral to incisive foramen, posterior to incisive foramen) described by Oliveira-Santos et al., ${ }^{11}$ were classified accordingly, in addition to the right and left emergence sides. For the sake of standardization, the axial sections were $0.25 \mathrm{~mm}$ thick (Figure 1).

\section{CS measurement}

The measurements of the CS location by the alveolar ridge were made using 1-mm-thick and 1-mm-long cross-sections, which were generated from the panoramic reconstruction created individually for each case (Figure 2). Once in possession of these cross-sections, after locating the CS, three measurements were carried out (Figure 3) as follows: a. NC (nasal cavity) - distance between the CS and the nasal cavity floor (red arrow);

b. BC (buccal cortical) - distance from the emergence of the CS to the buccal cortical bone edge, straight line (green arrow); and

c. RC (ridge crest) - distance from the emergence of the CS to the most prominent point of the crest of the alveolar ridge (blue arrow).

All classifications and measurements were performed by a dental surgeon who had a PhD in oral and maxillofacial radiology with extensive experience (those working for at least 4 years in the field) in handling CBCT images.

\section{Statistical analysis}

For the statistical analysis, three sample groups were used: FG - female group; MG - male group; and MXG - mixed group (both genders). Initially, a descriptive statistical analysis was carried out and absolute and relative numbers were reported. In addition to this first analysis, ANOVA (analysis of variance) was conducted in order to compare the right and left sides of the three measurements (RC; BC; NC) with a significance level of $95 \%$ $(p \geq 0.05)$ to check whether there were any differences between the sides. 


\section{Results}

\section{Female group}

The FG comprised a total of 284 patients, with the CS detected in 99 cases (34.86\%). Of the 99 cases, the CS was present only on the right side in 37 cases (37.37\%), only on the left side in 41 cases $(41.41 \%)$, and on both sides in 21 cases (21.21\%). The mean age (standard deviation) was 54.90 (11.87) when the CS was present and 53.98 (14.39) when it was absent.

In this group, according to Table 1 , there was no significant difference $(p=0.151)$ between the sides on which the CS was present as to the classification according to Oliveira-Santos et al., ${ }^{11}$ that is, a similar position was found between the right and left sides. The most common classification, both on the right and left sides, was location 5 - emergence of the CS behind

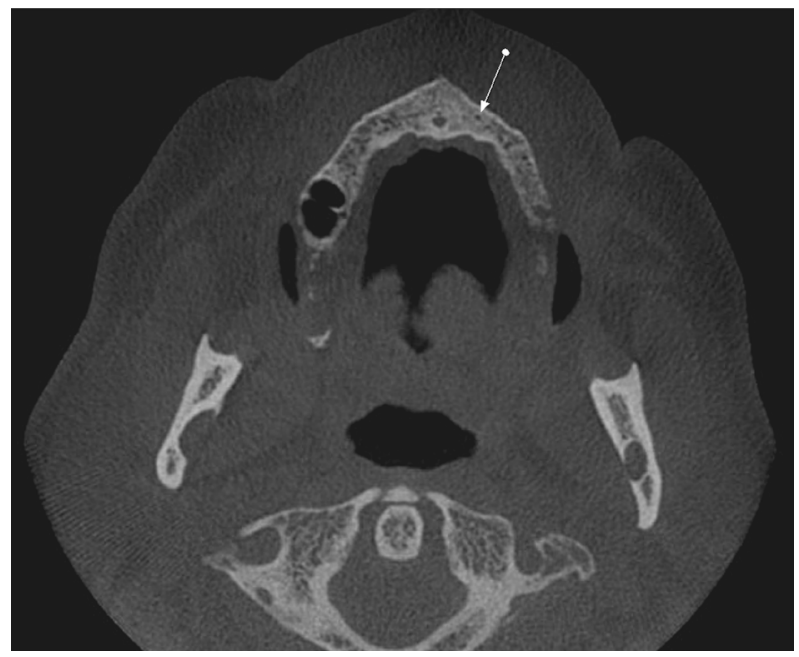

Figure 1. Axial CBCT showing the location of the CS behind the right canine (classification 6).

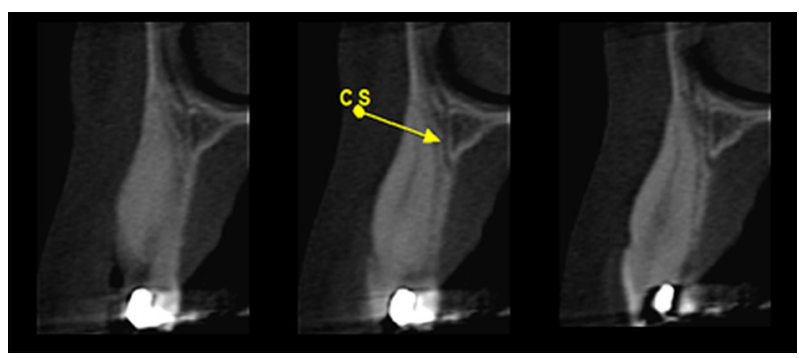

Figure 2. Example of $C S$ presence in $C B C T$ cross-sections in the 13th tooth region. the upper lateral incisor - which was present in 15 cases on the right side $(15.15 \%)$ and 14 on the left side (14.14\%). According to Table2, the following results were obtained for the $\mathrm{RC}, \mathrm{BC}$, and $\mathrm{NC}$ measurements, respectively: $p=0.104 ; p=0.047 ; p=0.606$. Therefore, there was a significant difference only in the BC measurement (i.e., from the CS to the ridge's buccal cortical bone).

\section{Male group}

The MG comprised a total of 216 patients, and the CS was present in 82 (37.97\%). The CS was present

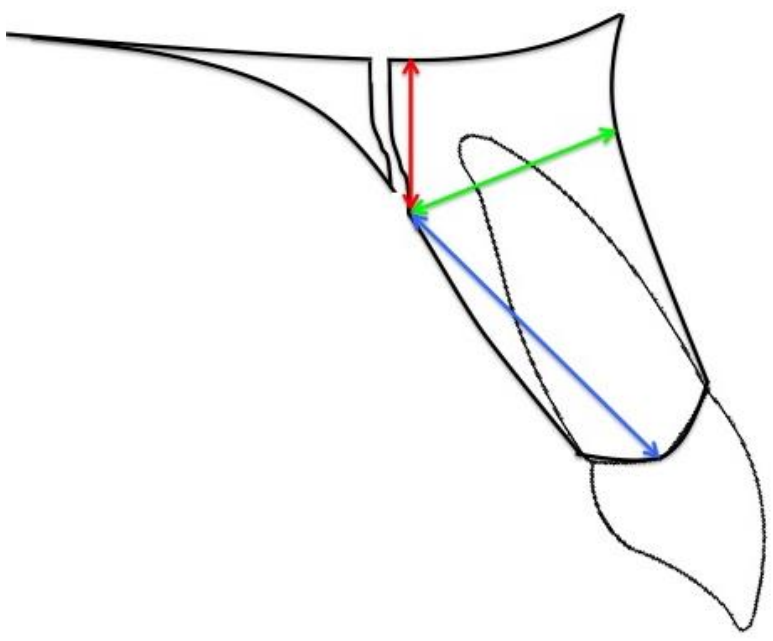

Figure 3. Measurements obtained between the CS and the reference structures in the cross-section of the anterior maxillary region: $\mathrm{NC}$ - red arrow, $\mathrm{BC}$ - green arrow, and $\mathrm{RC}$ - blue arrow.

Table 1. Absolute and relative numbers of location classification in accordance with Oliveira-Santos et al. 11 for female subjects.

\begin{tabular}{lcccc}
\hline \multirow{2}{*}{ Classification } & \multicolumn{2}{c}{ Right } & \multicolumn{2}{c}{ Left } \\
\cline { 2 - 5 } Absent & $\mathrm{n}$ & $\%$ & $\mathrm{n}$ & $\%$ \\
1 & 41 & 41.41 & 37 & 37.37 \\
2 & 2 & 2.02 & 2 & 2.02 \\
3 & 15 & 15.15 & 10 & 10.10 \\
4 & 7 & 7.07 & 8 & 8.08 \\
5 & 11 & 11.11 & 10 & 10.10 \\
6 & 15 & 15.15 & 14 & 14.14 \\
7 & 5 & 5.05 & 11 & 11.11 \\
Total & 3 & 3.03 & 7 & 7.07 \\
\hline $\mathrm{p}=0.1517$. & 99 & 100 & 99 & 100 \\
\hline & & & &
\end{tabular}


Table 2. Statistical data of the comparisons between the sides of measures RC, BC and NC for female subjects.

\begin{tabular}{lccccc}
\hline Side & Measure & $\mathrm{n}$ & Mean (SD) & Minimum & Maximum \\
\hline \multirow{3}{*}{ Right } & RC & & $7.06(5.31)$ & 1.20 & 24.22 \\
& BC & 58 & $6.29(3.31)$ & 1.00 & 16.50 \\
& NC & & $9.67(5.55)$ & 1.25 & 18.90 \\
Left & RC & & $8.80(6.28)$ & 1.12 & 23.27 \\
& BC & 62 & $7.54(3.58)$ & 1.51 & 18.20 \\
\hline
\end{tabular}

SD: standard deviation; RC: ridge crest; $\mathrm{BC}$ : buccal cortical; NC: nasal cavity.

Right X Left: RC $(p=0.104) ; C V(p=0.047) ; N C(p=0.606)$.

on both sides in 23 cases (28.05\%), on the right side in 27 cases $(32.93 \%)$, and on the left side in 32 cases (39.02\%). The mean age (standard deviation) was 56.16 (13.01) to 57.42 (11.96) when the CS was present and 55.38 (13.60) when it was absent.

There was no significant difference $(\mathrm{p}=0.215)$ in the classification of location between the sides when the CS was present, as noted in Table 3. The most frequent classification for both sides in this group was location 2 - beside the incisive foramen - with 12 cases on the right side (14.63\%) and 14 on the left side $(17.07 \%)$. The following results were obtained for the RC, BC, and NC measurements (Table 4): $p=0.253 ; p=0.139 ; p=0.516$. This demonstrates that there were no differences between the sides for any of the measurements.

\section{Mixed group}

There were a total of 500 patients in this group, and the CS was present in 181 (36.20\%). The CS was seen on both sides in 44 cases $(24.31 \%)$, on the right side in 64 (35.36\%), and on the left side in 73 (40.33\%). The mean age (standard deviation) was 55.11 (13.34) to 56.05 (11.95) when the CS was present and 54.57 (14.05) when it was absent.

In the classification of CS location, there was no significant difference $(p=0.055)$ between the sides, as shown in Table 5 . The most common location for both sides in this group was location 2 - beside the incisive foramen - with 27 cases on the right side $(14.92 \%)$ and 24 on the left side $(13.26 \%)$. It should be highlighted that, on the left side, location 5 - behind the upper lateral incisor - presented the same incidence as classification 2. The following results were obtained for the $\mathrm{RC}, \mathrm{BC}$, and $\mathrm{NC}$ measurements, respectively: $p=0.046 ; p=0.014$;
Table 3. Absolute and relative numbers of location classification in accordance with Oliveira-Santos et al. 11 for male subjects.

\begin{tabular}{lcccc}
\hline \multirow{2}{*}{ Classification } & \multicolumn{2}{c}{ Right } & \multicolumn{2}{c}{ Left } \\
\cline { 2 - 5 } & $\mathrm{n}$ & $\%$ & $\mathrm{n}$ & $\%$ \\
\hline Absent & 32 & 39.02 & 27 & 32.93 \\
1 & 3 & 3.66 & 1 & 1.22 \\
2 & 12 & 14.63 & 14 & 17.07 \\
3 & 7 & 8.54 & 6 & 7.32 \\
4 & 8 & 9.76 & 9 & 10.98 \\
5 & 11 & 13.41 & 10 & 12.20 \\
6 & 8 & 9.76 & 11 & 13.41 \\
7 & 1 & 1.22 & 4 & 4.88 \\
Total & 82 & 100 & 82 & 100 \\
\hline $\mathrm{p}=0.215$. & & & &
\end{tabular}

$p=0.583$, as shown in Table 6. Therefore, there was a difference in the distances from the CS to the alveolar ridge crest and to the buccal cortical bone in this group.

\section{Discussion}

Knowledge about anatomical variations is of extreme importance for treatment planning and post-treatment, as it can prevent complications during surgical procedures, ensuring a more favorable prognosis. ${ }^{1,2,3,5,14}$ Thus, it is necessary to know the anatomy of the area to be manipulated. This study had the CS, a branch of the infraorbital nerve, as its main focus. According to Rodella et al., ${ }^{3}$ the infraorbital nerve is an extension of the maxillary division of the trigeminal nerve.

The importance of this anatomical variation is highlighted when, in the rehabilitation of the anterior maxillary region for the placement of implants, the canine pillar is used as a definitive landmark for the support of implants, in which contact with the neurovascular bundle of the CS can compromise 
Location and classification of Canalis sinuosus for cone beam computed tomography: avoiding misdiagnosis

Table 4. Statistical data of the comparisons between the sides for measures RC, BC and NC for male subjects.

\begin{tabular}{lccccc}
\hline Side & Measure & $\mathrm{n}$ & Mean (SD) & Minimum & Maximum \\
\hline \multirow{3}{*}{ Right } & RC & & $8.46(5.48)$ & 1.46 & 18.75 \\
& BC & 50 & $7.46(3.28)$ & 1.50 & 13.69 \\
& NC & & $12.64(5.30)$ & 2.25 & 23.29 \\
Left & RC & & $9.81(6.52)$ & 1.50 & 13.69 \\
& BC & 55 & $8.98(3.15)$ & 2.25 & 17.76 \\
\hline
\end{tabular}

SD: standard deviation; RC: ridge crest; $\mathrm{BC}$ : buccal cortical; NC: nasal cavity.

Right X Left: RC $(p=0.104) ; C V(p=0.047) ; N C(p=0.606)$.

Table 5. Absolute and relative numbers of location classification in accordance with Oliveira-Santos et al.11 for the gathered grouped.

\begin{tabular}{lcccc}
\hline \multirow{2}{*}{ Classification } & \multicolumn{2}{c}{ Right } & \multicolumn{2}{c}{ Left } \\
\cline { 2 - 5 } Absent & $\mathrm{n}$ & $\%$ & $\mathrm{n}$ & $\%$ \\
\hline 1 & 73 & 40.33 & 64 & 35.36 \\
2 & 5 & 2.76 & 3 & 1.66 \\
3 & 27 & 14.92 & 24 & 13.26 \\
4 & 14 & 7.73 & 14 & 7.73 \\
5 & 19 & 10.50 & 19 & 10.50 \\
6 & 26 & 14.36 & 24 & 13.26 \\
7 & 13 & 7.18 & 22 & 12.15 \\
Total & 4 & 2.22 & 11 & 6.08 \\
\hline $\mathrm{p}=0.055$. & 181 & 100 & 181 & 100 \\
\hline
\end{tabular}

osteointegration and cause temporary or permanent paresthesia with bleeding in situ. $7,8,14$

The CS can be detected by preoperative imaging exams, especially computed tomography, which allow its visualization in thin sections without overlaps and also provide more reliable images of the maxillomandibular complex, given their 3D nature. This way, the use of CBCT enables the dentist to obtain the details needed for the diagnosis and treatment planning or surgery. ${ }^{12,13,15,16,17}$

The search for studies on the CS in the PubMed database detected only seven articles, four of which ${ }^{11,15,16,17}$ used CBCT for better evaluation of anatomical variation. The present study assessed 500 CBCT scans with the intention of locating the CS. Among the articles found in PubMed, the largest sample was that of Oliveira-Santos et al., ${ }^{11}$ who assessed CBCT scans of 178 subjects and found that 28 (15.7\%) had an additional foramen on the palate, with a total of 34 additional foramina being recorded. There were no statistical differences between the patients either with or without an additional foramen on the palate when compared by gender or age, which is not in agreement with the findings of the present study because when all the patients (mixed group) were assessed, there was a variation between the right and left sides regarding the distances between the CS and the alveolar bone crest and between the CS and the buccal cortical bone. This can be explained by the fact that the alveolar bone plate is subjected to morphological changes over time. In addition, this group showed a greater number of patients $(n=500)$, which resulted in greater variations. In the FG, there was a difference between the right and left sides only in the distance between the CS and the cortical bone, which is justified by the same reason described for the MG. It should be underscored that neither the traumas to which the bone plate was submitted nor the patient's dental condition indicating toothlessness, surgeries, or trauma in the region were considered in this study. The reason for evaluating the MXG is that there is sexual dimorphism for some anatomical variations; however, our results show that gender does not interfere with variations of the CS, although variations have been found between the right and left sides in the distances between the CS and the alveolar bone crest and between the CS and the buccal cortical bone, as pointed out earlier.

Wanzeler et al. ${ }^{16}$ assessed 100 CBCT images obtained from a dental clinic in the city of Belém, Pará, Brazil, in order to identify the location of bifurcation, CS diameter, and the direction of its course and distance to the alveolar bone crest. The CS was present in $88 \%$ of the sample. There were no statistically significant changes in the presence of the CS related to gender, age, and course 
Table 6. Statistical data of the comparisons between the sides for measures RC, BC and NC for the gathered grouped.

\begin{tabular}{|c|c|c|c|c|c|}
\hline Side & Measure & $n$ & Mean (SD) & Minimum & Maximum \\
\hline \multirow{3}{*}{ Right } & $\mathrm{RC}$ & & 7.71 (5.41) & 1.20 & 24.22 \\
\hline & $B C$ & 108 & $6.83(3.34)$ & 1.00 & 16.50 \\
\hline & NC & & $11.05(5.61)$ & 1.25 & 23.29 \\
\hline \multirow{3}{*}{ Left } & $\mathrm{RC}$ & & $9.28(6.38)$ & 1.12 & 23.27 \\
\hline & $B C$ & 117 & 7.94 (3.39) & 1.51 & 18.20 \\
\hline & NC & & $10.44(5.44)$ & 1.85 & 24.40 \\
\hline
\end{tabular}

SD: standard deviation; RC: ridge crest; $\mathrm{BC}$ : buccal cortical; NC: nasal cavity; CS: canalis sinuosus.

Right X Left: $C S-$ Crest $(p=0.046)$; CS - Plate $(p=0.014)$; $C S$ - Floor $(p=0.583)$.

direction. The authors concluded that the CS was frequent and similar in both genders, with a distant course to the alveolar bone crest ending in different locations, which is consistent with the present study, suggesting that the CS should be analyzed using CBCT before performing surgical procedures. von Arx et al. ${ }^{17}$ evaluated $176 \mathrm{CBCT}$ images and found the CS in 49 patients (27.8\%). The greatest canal frequency was observed more often in male $(33.0 \%)$ than in female $(22.7 \%$; $p=0.130)$ subjects, though gender and age did not significantly influence CS diameter. The authors concluded that, from a clinical perspective, further studies are needed to determine the content of these canals.

The results of this paper are in agreement with those of previous studies ${ }^{11,13,15,16,17,18}$ that pointed out the need of CBCT scans for identifying anatomical variations, allowing three-dimensional imaging, detailed assessments, and proper treatment

\section{References}

1. Jones FW. The anterior superior alveolar nerve and vessels. J Anat. 1939;73(Pt 4):583-91.

2. Shelley AM, Rushton VE, Horner K. Canalis sinuosus mimicking a periapical inflammatory lesion. $\mathrm{Br}$ Dent J. 1999 Apr 24;186(8):378-9. Erratum in Br Dent J. 1999 Jun 12;186(11):550.

3. Rodella LF, Buffoli B, Labanca M., Rezzani R. A review of the mandibular and maxillary nerve supplies and their clinical relevance. Arch Oral Biol. 2012;57(4):323-34. doi:10.1016/j.archoralbio.2011.09.007

4. Neves FS, Cruzoé-Souza M, Franco LCS, Caria PHF, Bonfim-Almeida P, Cruzoé-Rebello I. Canalis sinuosus: a rare anatomical variation. Surg Radiol Anat. 2012;34(6):563-6. doi:10.1007/s00276-011-0907-6.

5. Borbolato RM, Ambiel CR. Trigeminal neuralgia: important aspects in dental practice. Rev Saude Pesqui. 2009;2(2):201-8. Portuguese. planning. Oliveira-Santos et al. ${ }^{11}$ also highlighted that the foramen and canals in the anterior superior maxillary region are relatively frequent and that dental surgeons must be aware and well trained to identify these variations.

This study reaffirms the need for CBCT before carrying out invasive procedures, seeking to preserve noble anatomical structures in the anterior maxillary region, as it is in the canine pillar region, which is a frequent location of rehabilitating implant procedures where there is a higher incidence of CS emergence.

\section{Conclusion}

According to the results obtained here, it may be concluded that there is a variation in the location of the CS if compared to the crest and buccal cortical bone of the ridge, assuming that it is going to be located by the upper lateral incisor palatine.

6. Sedentexct. Provisional guidelines on CBCT for dental and maxillofacial radiology [Internet]. Manchester: University of Manchester; 2016 [cited 2011 Apr 1]. Available from: http://www.sedentexct.eu/system/ files/sedentexct_project_provisional_guidelines.pdf

7. Faria CAR, Barros RA, Modesto D, Navarro JAC. Anatomical variation of the infra-orbital plexus. Rev Bras Otorrinolaringol. 1981;47(2):161-5. Portuguese.

8. Brandão FH, Machado MRCS, Aquino JEP, Coelho Júnior RG, Pereira SHP, Fabi RP. The Foramen and infraorbital nerve relating to the surgery for external access to the maxillary sinus. Arq Int Otorrinolaringol. 2008;12(3):342-6. Portuguese.

9. Correia F, Salgado A. Cone beam computed tomography and its application in dental practice. Rev Port Estomatol Cir Maxilofac. 2012;53(1):47-52. Portuguese. 
10. Ueki K, Hashiba Y, Marukawa K, Nakagawa $\mathrm{K}$, Okabe K, Yamamoto E. Determining the anatomy of the descending palatine artery and pterygoid plates with computed tomography in Class III patients. J Craniomaxillofac Surg. 2009;37(8):469-73. doi:10.1016/j.jcms.2009.03.010

11. Oliveira-Santos C, Rubira-Bullen IRF, Monteiro SAC, Léon JE, Jacobs R. Neurovascular anatomical variations in the anterior palate observed on CBCT images. Clin Oral Implants Res. 2013;24(9):1044-8. doi:10.1111/j.1600-0501.2012.02497.x

12. Oliveira-Santos CO, Souza PH, Berti-Couto SA, Stinkens L, Moyaert K, Van Assche N, et al. Characterisation of additional mental foramina through cone beam computed tomography. J Oral Rehabil. 2011;38(11):595-600. doi:10.1111/j.1365-2842.2010.02186.x

13. Temmerman A, Hertele S, Teughels W, Dekeyser C, Jacobs R, Quirynen M. Are panoramic images reliable in planning sinus augmentation procedures? Clin Oral Implants Res. 2011;22(2):189-94. doi:10.1111/j.1600-0501.2010.02000.x
14. Olenczak JB, Hui-Chou HG, Aguila DJ 3rd, Shaeffer CA, Dellon AL, Manson PN. Posttraumatic midface pain: clinical significance of the anterior superior alveolar nerve and canalis sinuosus. Ann Plast Surg. 2015;75(5):543-7. doi:10.1097/SAP.0000000000000335.

15. Torres MGG, Valverde LF, Vidal MTA, Crusoé-Rebello IM. Branch of the canalis sinuosus: a rare anatomical variation - a case report. Surg Radiol Anat. 2015;37(7):879-81. doi:10.1007/s00276-015-1432-9

16. Wanzeler AM, Marinho CG, Alves Junior SM, Manzi FR, Tuji FM. Anatomical study of the canalis sinuosus in 100 cone beam computed tomography examinations. Oral Maxillofac Surg. 2015;19(1):49-53. doi:10.1007/s10006-014-0450-9

17. von Arx T, Lozanoff S, Sendi P, Bornstein MM. Assessment of bone channels other than the nasopalatine canal in the anterior maxilla using limited cone beam computed tomography. Surg Radiol Anat. 2013;35(9):783-90. doi:10.1007/s00276-013-1110-8.

18. Shelley AM, Rushton VE, Horner K. Canalis sinuosus mimicking a periapical inflammatory lesion. $\mathrm{Br}$ Dent J. 1999 Apr 24;186(8):378-9. Erratum in Br Dent J 1999 Jun 12;186(11):550. 